\title{
Real Microstructure Modeling for Stiffness and Strength Analy- ses of Texture in Ores
}

\author{
Tatsuhiko AIZAWA, Yoshihiro SUWA ${ }^{1)}$ and Shinji MURAISHI ${ }^{21}$ \\ Center for Collaborate Research, The University of Tokyo, 4-6-1 Komaba, Meguro-ku, Tokyo 153-8904 Japan. \\ 1) Research Center for Advanced Science and Technology, The University of Tokyo, 4-6-1 Komaba, Meguro-ku, Tokyo 153-8904 Japan. \\ 2) Department of Materials Science and Technology, Tokyo Institute of Technology, 2-12-1 Ookayama, Meguro-ku, Tokyo 152- \\ 8552 Japan.
}

(Received on May 6, 2004; accepted in final form on July 3, 2004)

\begin{abstract}
Theoretical model is proposed to make stiffness and strength analyses for various multi-phase, porous media including the sintered ores. On the basis of the multilevel modeling, a macro-model is used to estimate the crushing strength of sintered ore with various compositions of texture. A micro model for each texture is constructed to represent its characteristic microstructure and to make stiffness and strength analyses. Since the real microstructure is taken into account of the unit cell, the homogenized stiffness provides us the elastic properties of textures with or without micro-pores. The stress analysis both for texture and crushing test-specimen, provides us the theoretical estimate of fracture strength, respectively. Three typical textures in the sintered ore are employed for application of the present theoretical models. The procedure to deduce the theoretical model from the optical micrograph is described for the texture of acicular calcium ferrite. Using the unit cell models for each texture, which is objective to the cell size and geometry, the stiffness and fracture strength are estimated. Assuming that the sintered ore specimen is composed of two phases or composite of porous hematite and slag-hematite complex, the fracture strength is also estimated as the function of volume fraction for porous hematite.
\end{abstract}

KEY WORDS: porous medium; sintered ore; theoretical model; multilevel modeling; unit cell; real microstructure; homogenized stiffness; fracture strength; texture; crushing test.

\section{Introduction}

Sintered ore is a typical porous medium, which is difficult to evaluate its strength and stiffness for reliability and integrity assurance in practice. The first difficulty lies in the geometrical complexity, where oxides and melts are distributed together with various types of pores and porosities. ${ }^{1)}$ Typical cross-sectional micrograph is shown in Fig. 1. Large pores are located in the sinter while many porosities are left in the matrix. Significant deviation of strength and stiffness from the average values is driven by this porosity distribution. In the second, the matrix of sinter is composed of many phases with different geometry and microstructure. Besides the hematite, the calcium ferrite (CF) or slags are distributed together with pores in the sinter, so that the strength and stiffness of the whole sinter cannot be estimated without mechanical information of each texture. Although the crushing load of sintered specimens is experimentally measured, its dependency on the porosity and texture is never fully understood, leaving many unknowns open to discussion. ${ }^{2)}$

Authors have developed the multilevel modeling to make strength and stiffness analysis of porous solids and cellstructured materials. ${ }^{3-5)}$ This method has two main characteristic features: hierarchical structure and coupled analysis. In the former, the macro-model for deformation and stress analyses of specimens and structural components is com- bined in the hierarchical manner with a micro-model for physical description of microstructure evolution. Hot deformation and sintering behavior of porous materials is analyzed even without their constitutive equations. In the latter, multi-physical models are coupled into the hierarchical modeling. Hot deformation of materials is analyzed together with the thermal analysis to simulate the thermal tran-

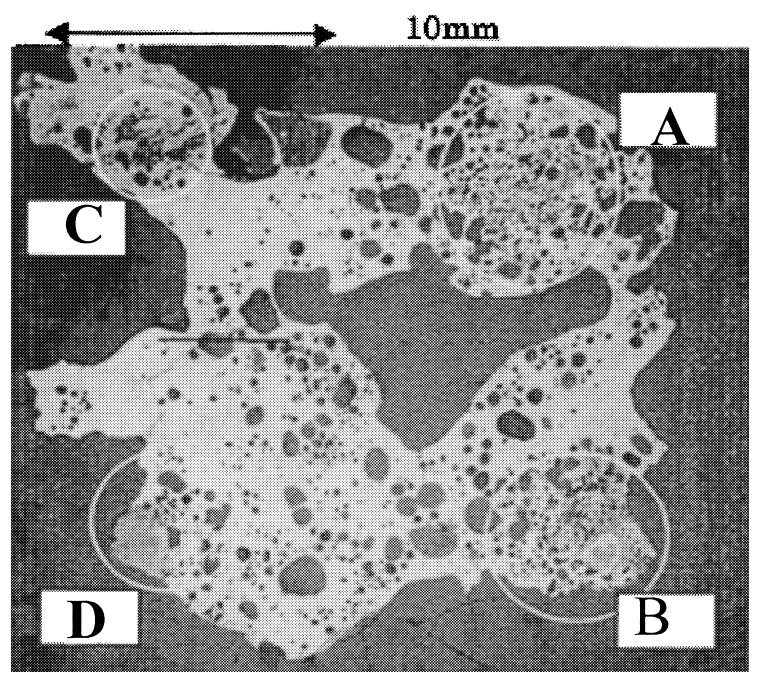

Fig. 1. Typical, cross-sectional microstructure of the sintered ore; four domains from "A" to "D" were selected to search for textures. 
sients. ${ }^{6)}$ Generation and propagation of voids in creep and creep/fatigue behavior is coupled with the hot deformation analyses. $^{7,8)}$

In the present paper, the real microstructure modeling is proposed for strength and stiffness analyses of sintered ores on the basis of the multilevel modeling. In this approach, two models are utilized in the hierarchical manner for theoretical formulation. The micro-model is used to make stress analysis in the unit cell, which is locally selected as a representative region of microstructure. Since this unit cell is determined in the objective manner to size and geometry, the calculated mechanical quantities and properties can be utilized as the true stress and strain distribution in the selected microstructure and as its equivalent elastic moduli. First, the fundamental frame of the real microstructure modeling is introduced together with comments on the essential formulation in the homogenized computational mechanics. Typical textures of the sintered ore are employed to construct their unit cell models and to make their stiffness and strength analyses. Their fracture strength is estimated by assuming the criterion of limit strain. The macro-model is used together with micro models to evaluate the crushing strength of the sintered-ore specimen. Assuming that the specimen is composed of two-phase mixture, the sensitivity of fracture strength to the volume fraction of either texture is estimated to describe the crushing behavior of the sintered ore.

\section{Real Microstructure Modeling}

Fundamental theories of the homogenization theory as well as their extension to computational mechanics were seen elsewhere. ${ }^{9-11)}$ The essential formulation is only briefly stated in what follows. The present modeling is based on the hierarchical frame of macro- and micro-models as shown in Fig. 2. $X$-coordinates describe the deformation of macro-model while $Y$ denotes for coordinates of the micromodel. Assuming that a unit cell with the periodicity of $\varepsilon$ is representative of the local region in the macro-model, the whole displacement $u(x, y)$ is represented by the asymptotic expansion. If $\varepsilon$ is sufficiently small, this expansion is approximated by the sum of the average displacement $u^{0}(x)$ and the local disturbance $u^{1}(x, y)$ :

$$
u(x, y) \approx u^{0}(x)+\varepsilon u^{1}(x, y)
$$

Here, $Y$-periodicity is assumed to hold on. Then

$$
\frac{\partial}{\partial x_{j}} u_{i}(x, y)=\frac{\partial u_{i}}{\partial x_{j}}+\frac{1}{\varepsilon} \frac{\partial u_{i}}{\partial y_{j}}
$$

and

$$
\int_{V} u_{i}(x, y) d V=\int_{V} \frac{1}{|Y|}\left(\int_{Y} u_{i}(x, y) d Y\right) d V
$$

where $V$ is the volume of macro-model and $Y$, the volume of micro-model. Under this assumption, the variational equation in $V$ is equivalent to the system of variational equations both for macro- and micro-models. Solution of each variational equation for each model provides both $u^{0}$ and $u^{1}$. Then, the whole deformation, strain and stress states

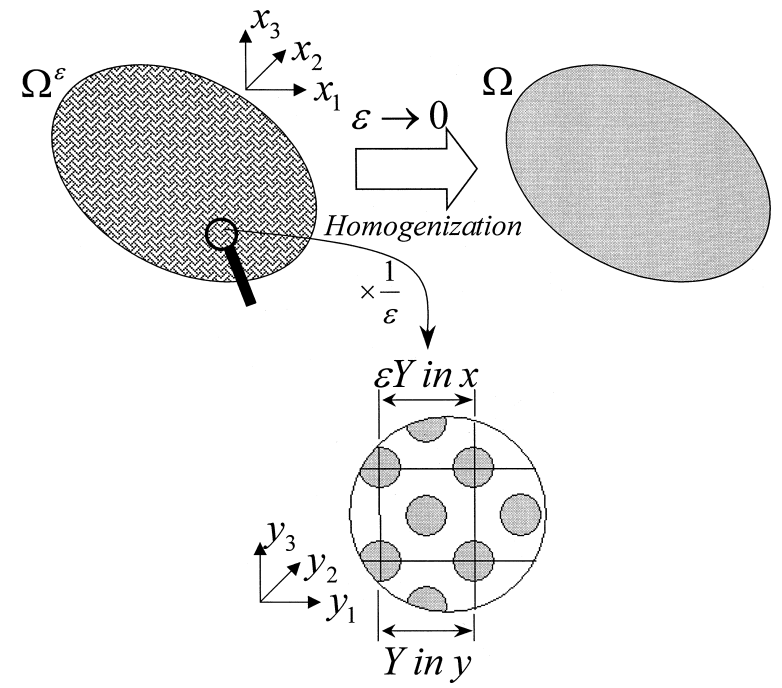

Fig. 2. Relationship between macro- and micro-models in the multilevel modeling

are uniquely determined. In this homogenized computational mechanics, the unit cell can be arbitrary selected if the calculated mechanical properties are completely free from the periodicity of $\varepsilon$. This implies that real microstructure observed by SEM or optical microscope should be taken into account by unit cell selection.

In the solution of the variational equation for the micromodel, physical properties of constituent materials are indispensable. That is, the mechanical response in the unit cell is precisely analyzed to provide the local displacement, strain and stress distributions. While the homogenized properties, which are used in the solution of the variational equation for the macro-model, are deduced by the micromodel. For an example, the homogenized stiffness tensor $D_{i j k l}^{\mathrm{H}}$ for the macro-model is uniquely provided by

$$
D_{i j k l}^{\mathrm{H}}=\frac{1}{|Y|} \int_{Y}\left(D_{i j k l}-D_{i j \mathrm{pq}} \frac{\partial \chi_{\mathrm{p}}^{k l}}{\partial y_{\mathrm{q}}}\right) d Y
$$

where $\chi$ is the characteristic function in the micro-model. This function is calculated together with computation of $u^{1}(x, y)$. This stiffness tensor is dependent not only on the geometry and microstructure of unit cell but also on the strain distribution in the unit cell.

In the present paper, a unit cell is selected in the adaptive way to the real microstructure for stiffness and stress analyses. In the case of the polycrystalline materials, both the intra-grain regions and the grain boundaries, must be identified as a separate group in the theoretical model. As shown in Fig. 3, the procedure to construct the theoretical model is consisted of three stages. At first, the original micrograph is image-processed to make registration of intragrains and grain-boundaries. In the half-tone image, these two regions are recognized to have different pixel values and each intragrain and grain boundary is segmented. In the second stage, a unit cell is determined to be representative of this microstructure. As before mentioned, the unit cell size or periodicity must be selected to have mechanical properties indifferent to the cell size. Then, in the third stage, this selected unit cell is discretized to a theoretical model to be directly used for computation. The finite ele- 


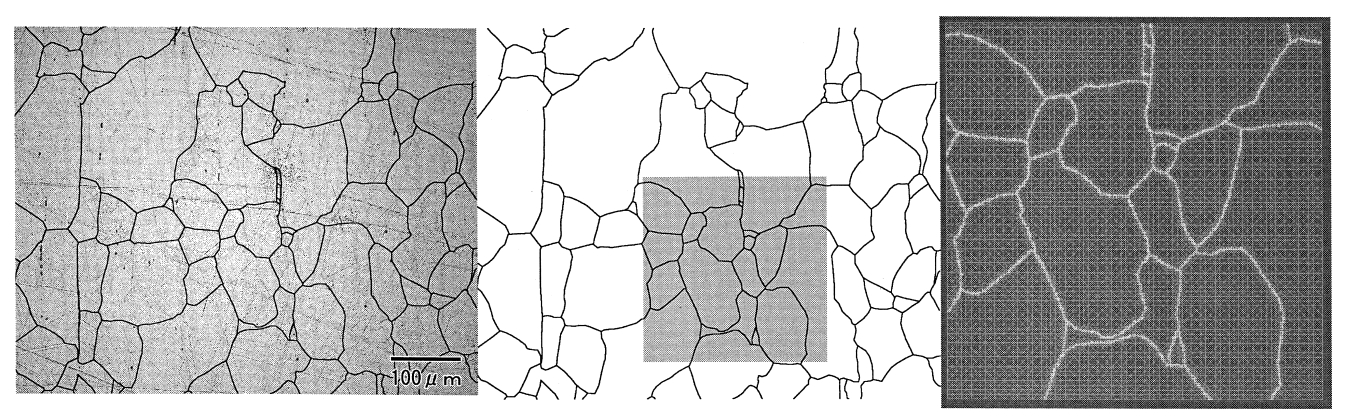

Fig. 3. Procedure to construct the real microstructure from the optical and SEM micrographs.
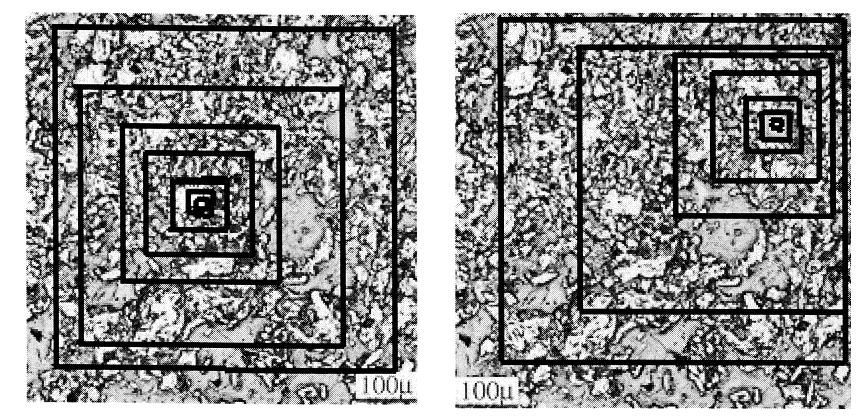

Fig. 4. Automatic segmentation to determine the objective unit cell size.

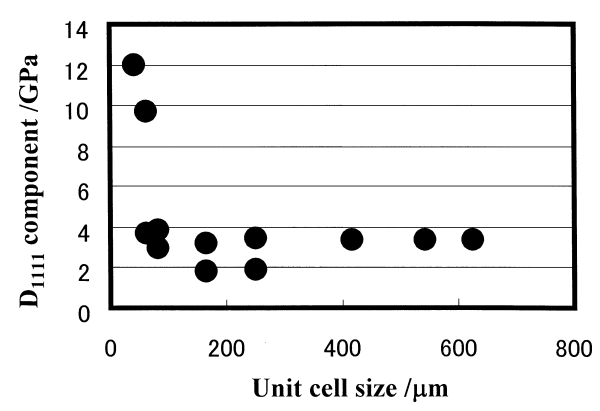

Fig. 5. Convergence of the homogenized stiffness with enlarging the unit cell size.

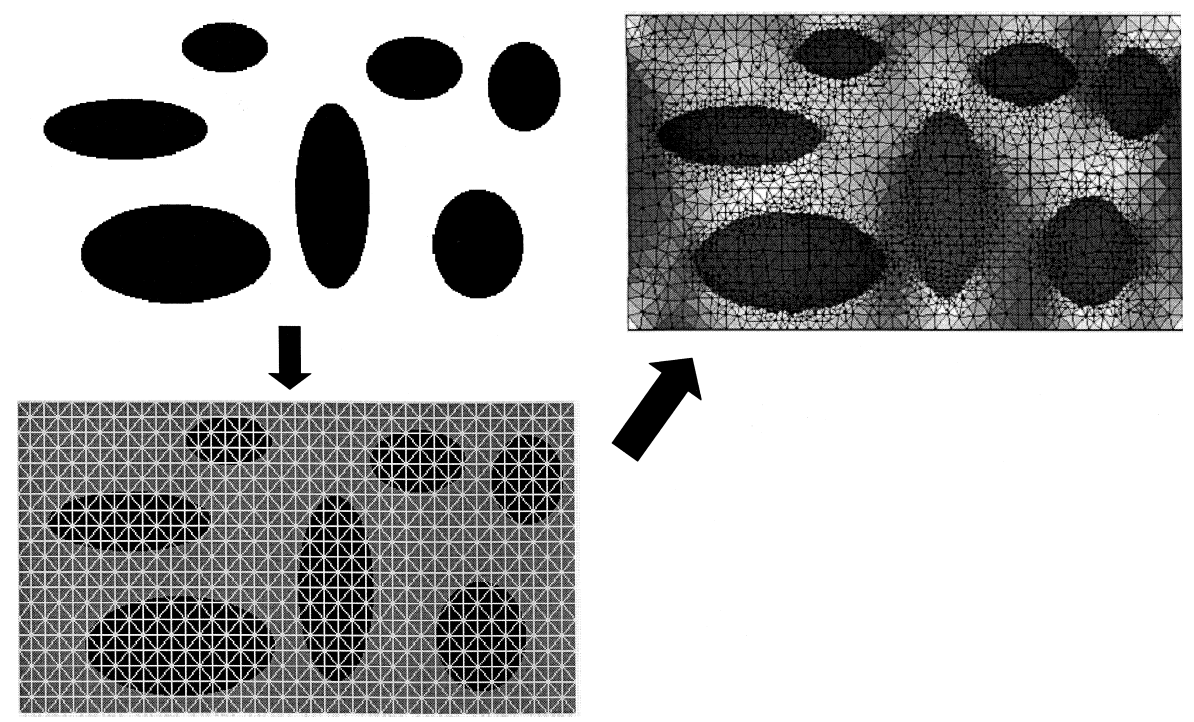

Fig. 6. Automatic generation of theoretical model.

ments each for intragrain and grain boundary are also segmented to represent the stress and strain state in each region by post-processing.

In the above second stage, the unit cell size is automatically changed to generate the mathematical models and to calculate the homogenized stiffness tensor in the function of the unit cell size. Figure 4 depicted typical searching for an objective unit cell by automatically changing the position and size. In general, if the unit cell size is selected too small to represent the microstructure adequately, the calculated stiffness has significant deviation around the average. As shown in Fig. 5, with increasing the unit cell size, this deviation is reduced to null in order that the homogenized stiffness should be indifferent to the geometric configuration. That is, objective unit cell can be selected to have unique mechanical properties to each microstructure.

In the third stage, the selected unit cell is automatically transformed into an assembly of finite elements. Consider the case where several inclusions are embedded in the matrix in Fig. 6. Both nodes and elements are regularly generated in the unit cell. With consideration of interfaces between different phases of materials, the original positions of nodes are shifted to preserve the continuity of displacement across their interface. As before mentioned, both inclusions and matrix are represented by the specified groups of elements. Their interfacial boundaries are also represented by a series of nodes. Hence, after stiffness and stress analysis, displacement, strain and stress state on each region or boundary is reproduced by archiving process. Through the above three stages, real microstructure modeling provides 

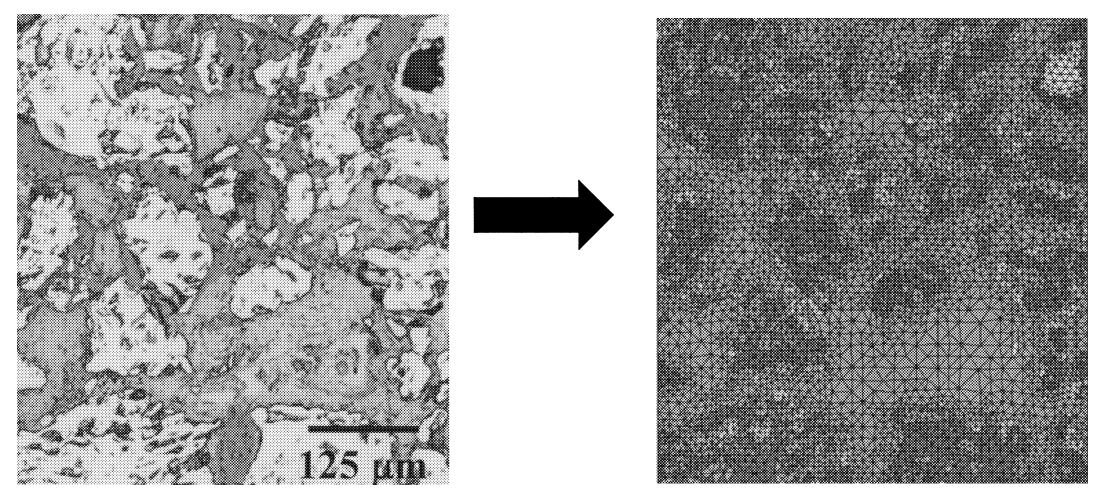

Fig. 7. Typical generation of theoretical model from the optical micrograph for the sintered ore.

us the objective unit-cell model from the SEM and optical micrographs. Figure 7 compared an original unit cell of a sintered ore and the corresponding theoretical model.

In order to verify the reliability of this theoretical approach, the homogenized stiffness of porous hematite was calculated and compared with the experimental data. ${ }^{12)}$ In this computation, the Young's modulus is directly estimated from $D_{i j k l}^{\mathrm{H}}$ by the equation. ${ }^{4)}$ While in experiments, the Young's modulus was measured from the gradient in the load-displacement curves. Dense hematite is assumed to have the Young's modulus by $E=181 \mathrm{GPa}$ and the Poisson's ratio by $v=0.3$. Figure 8 compared both results. Two types of mechanical state were assumed in this two-dimensional computation: plain strain and plain stress conditions. Irrespective of this assumption, both experimentally measured Young's modulus and the homogenized stiffness are in fairly good agreement. This implies that the homogenized stiffness is equivalent to the actual elastic properties of porous media once the unit cell is objectively selected by the present procedure.

\section{Stiffness and Strength Analysis of Textures in Ores}

The theoretical model is constructed to be representative of the microstructure from the micrograph of texture. Both stiffness and stress analyses for each texture, are made by using the theoretical models.

\subsection{Computational Mechanics Modeling of Textures}

Figure 9 illustrated the standard procedure to construct the theoretical model from the optical micrograph for the selected texture. First, the original micrograph is segmented to an image including the texture. In this case, acicular calcium ferrite is chosen as a targeting texture. Using the image processing, the segmented model has only a single phase of texture. In this stage, the region with the targeting texture is selected to have the specified concentration in the half-tone image, exclusive of other phases. After registration of acicular CF phase in the image, other phases are assigned to be porosity or other materials. In this case, other regions than acicular $\mathrm{CF}$, are assumed to be composed of a matrix, which has lower stiffness and strength than $\mathrm{CF}$ for convenience of computational modeling. In the same way as stated in Fig. 4, the unit cell size is automatically determined and subsequently is further discretized to the theoretical model. Figure 10 compared three types of theoretical models, which are respectively representative of the porous

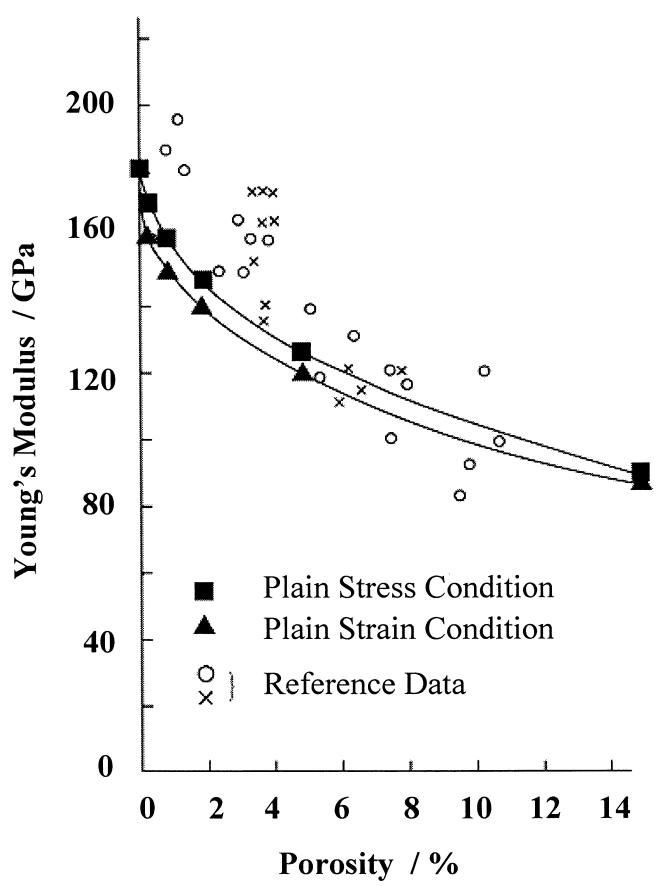

Fig. 8. Evaluation of Young's modulus for porous hematite with comparison between the present results and the experimental data.

hematite, the acicular calcium ferrite and slag-hematite complex. These three models are directly used for stiffness and stress analysis of texture.

\subsection{Stiffness Analysis}

The elastic properties of each constituent material for texture are necessary in computation. Table $\mathbf{1}$ listed the elastic properties of hematite, matrix and slag as a single phase without pores. The strength of each phase was estimated from the hardness. Table 2 compared the calculated components of homogenized stiffness tensor in the plain strain condition by using Eq. (4). Young's moduli along each coordinate direction or $\left\{E_{11}, E_{22}\right\}$, are defined from $D_{i j k l}$ by

$$
\begin{aligned}
& E_{11}=D_{1111}-\left(D_{1212}\right)^{2} / D_{2222}, \\
& E_{22}=D_{2222}-\left(D_{1212}\right)^{2} / D_{1111} \ldots
\end{aligned}
$$

Then, the average of Young's modulus for each unit cell, or, $E_{\text {ave }}$, is estimated by $\left(E_{11}+E_{22}\right) / 2$. Since most of unit cells for texture have a little crystallographic anisotropy, there might be a slight difference among $E_{11}, E_{22}$ and $E_{\text {ave }}$. 

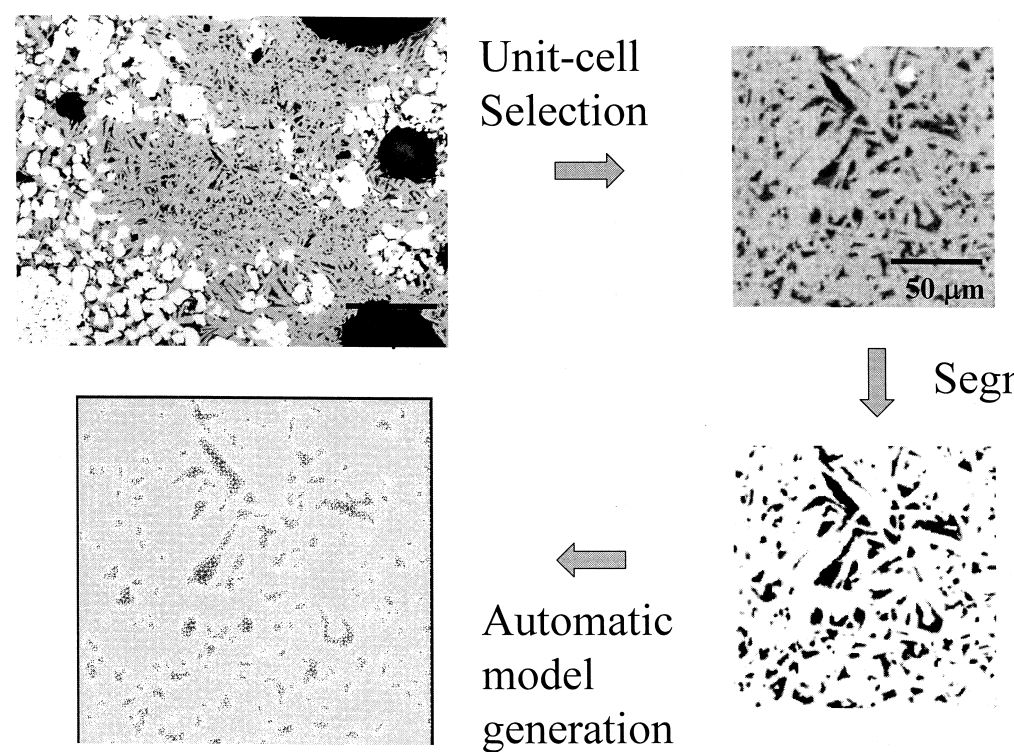

Fig. 9. Generation of theoretical model for the selected texture from the optical micrograph.
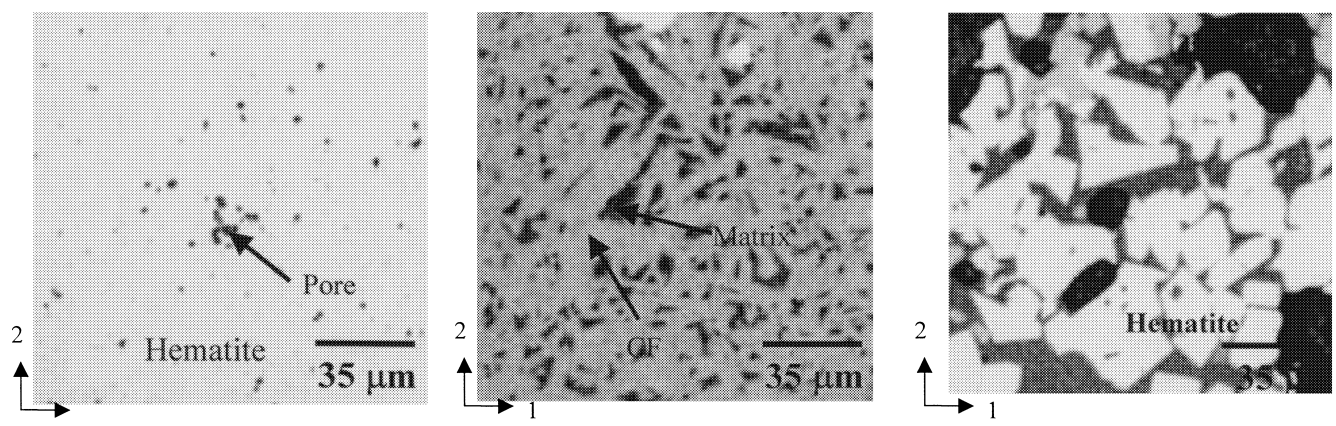

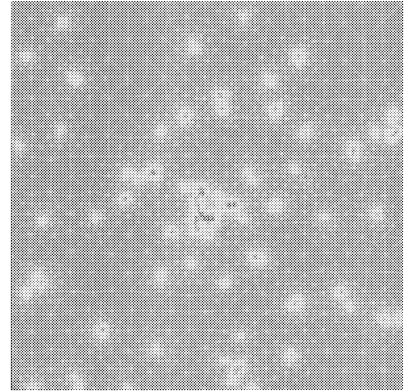

a) Porous hematite

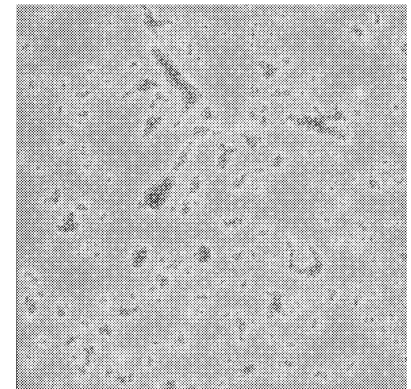

b) Acicular CF

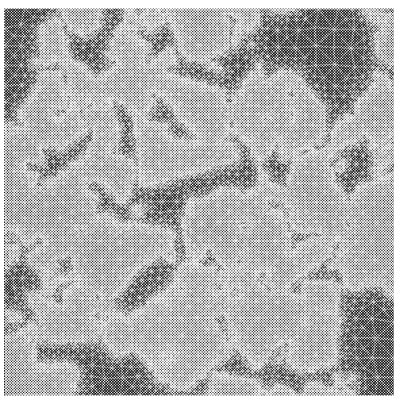

c) Slag-Hematite Complex

Fig. 10. Three types of theoretical models generated from the optical micrograph including each texture: a) Porous hematite, b) acicular calcium ferrite and c) slag-hematite complex.

Table 1. Elastic properties of constituent materials in the sintered ore.

\begin{tabular}{|l|c|c|c|}
\hline Constituents & $\begin{array}{c}\text { Young's Modulus } \\
(\mathrm{E})\end{array}$ & $\begin{array}{c}\text { Poisson's Ratio } \\
(v)\end{array}$ & Ultimate Strength \\
\hline Hematite & $177 \mathrm{GPa}$ & 0.24 & $262 \mathrm{MPa}$ \\
\hline Calcium Ferrite & $245 \mathrm{GPa}$ & 0.3 & $362 \mathrm{MPa}$ \\
\hline Matrix & $25 \mathrm{GPa}$ & 0.3 & $40 \mathrm{MPa}$ \\
\hline Slag & $93 \mathrm{GPa}$ & 0.3 & $135 \mathrm{MPa}$ \\
\hline
\end{tabular}

\subsection{Strength Analysis}

Various loading and boundary conditions can be taken into account in the stress analysis. In this computation, the stress and strain distribution of materials under the uni-
Table 2. Comparison of the homogenized stiffness tensor among three textures.

\begin{tabular}{|l|c|c|c|c|}
\hline Texture & $\begin{array}{c}\mathrm{D}_{1111} \\
(\mathrm{GPa})\end{array}$ & $\begin{array}{c}\mathrm{D}_{2222} \\
(\mathrm{GPa})\end{array}$ & $\begin{array}{c}\mathrm{D}_{1212} \\
(\mathrm{GPa})\end{array}$ & $\begin{array}{c}\mathrm{E}_{\text {ave }} \\
(\mathrm{GPa})\end{array}$ \\
\hline Porous Hematite & 180 & 180 & 43 & 169 \\
\hline $\begin{array}{l}\text { Acicular } \\
\text { Calcium Ferrite }\end{array}$ & 33.2 & 33.4 & 0.99 & 30.3 \\
\hline $\begin{array}{l}\text { Slag-Hematite } \\
\text { Complex }\end{array}$ & 149 & 149 & 45.3 & 135 \\
\hline
\end{tabular}

formly applied compressive strain is obtained by using the theoretical models. The periodic boundary conditions were assumed both on the right and left sides of a unit cell. The 

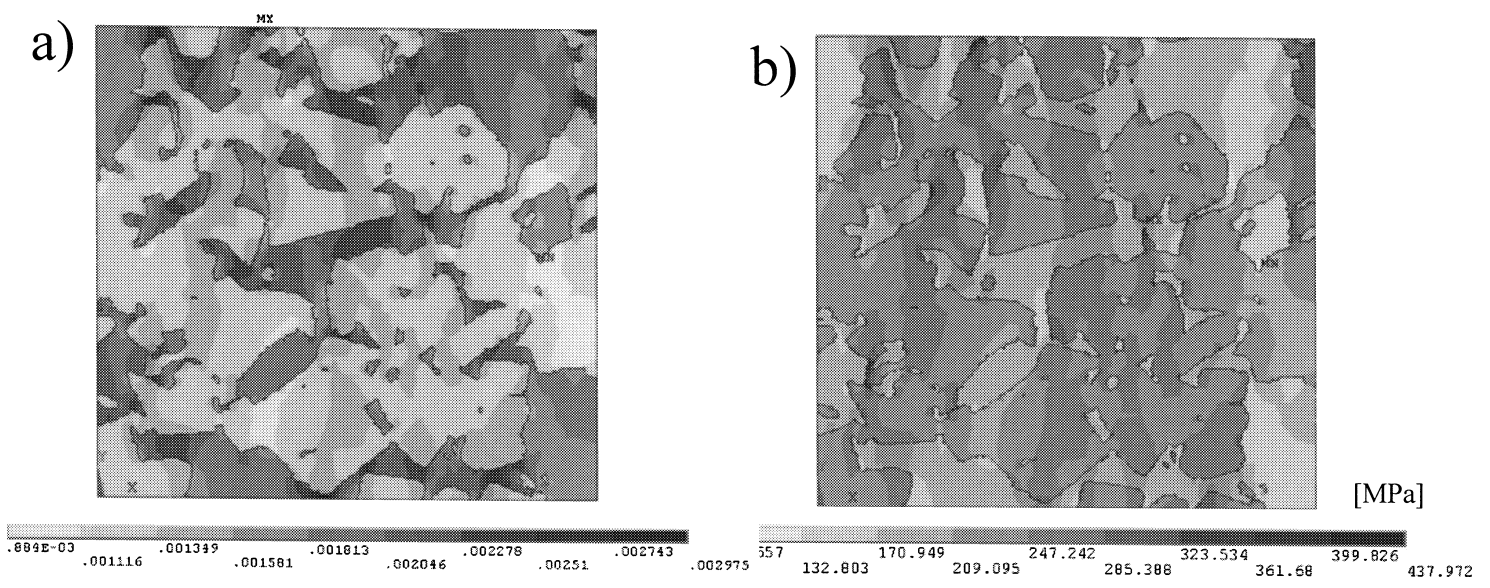

Fig. 11. The calculated stress and strain distributions in the slag-hematite complex under the uniform compressive strain: a) Equivalent strain distribution and b) equivalent stress distribution.

vertical displacement along its lower side was fixed while the horizontal one was free. On its upper side, the vertical displacement was prescribed by a uniform value. Figure 11 depicted the calculated stress and strain distributions by using the unit cell model of Fig. 10c) for the slag-hematite complex. Even when the same uniform strain is applied to both phases, the stress concentrates in the phase of slag since it has higher stiffness than the hematite.

\section{Discussion}

In the developed theoretical model, stress and strain information is post-processed to estimate the fracture stress of selected texture and sintered-ore specimen.

\subsection{Post-processing}

The present unit cell model has attributes of materials for segmentation of models. As shown in Fig. 12, the region either for hematite or for slag is selected to depict the stress distribution in each selected material. Local region with maximum strain or stress can be deduced by archiving the strain and stress distributions. In the following, the position with maximum strain is searched in the theoretical model to calculate the strain concentration factor $(\alpha)$. First, local strain distribution with maximum strain is searched by the above archiving procedure. Instead of point-wise maximum, the locally averaged maximum strain $\left(\varepsilon_{\max }\right)$ is calculated by using the local strain distribution. Then, $\alpha$ is defined in each stress/strain analysis by the ratio of $\varepsilon_{\max }$ to the uniformly applied strain.

\subsection{Estimate of Fracture Strength for Texture}

Among several criteria of fracture for brittle materials, the limit strain is employed as a criterion. That is, the brittle material fractures when the local maximum strain reaches the limit strain $\left(\varepsilon_{\text {limit }}\right)$. For simplicity, $\varepsilon_{\text {limit }}$ is given by the off-set strain of $0.2 \%$ : i.e., $\varepsilon_{\text {limit }}=0.002$. Then, the fracture stress $\left(\sigma_{\mathrm{F}}\right)$ is estimated by $\sigma_{\mathrm{F}}=E_{\text {ave }}\left(\varepsilon_{\text {limit }} / \alpha\right)$, where $E_{\text {ave }}$ is the averaged Young's modulus, calculated from the homogenized stiffness tensor by Eq. (5). Table 3 listed the position with maximum strain in the texture model and the fracture stress. This reveals that fracture stress of each texture can be theoretically estimated once the microstructure of texture is obtained in experiment.

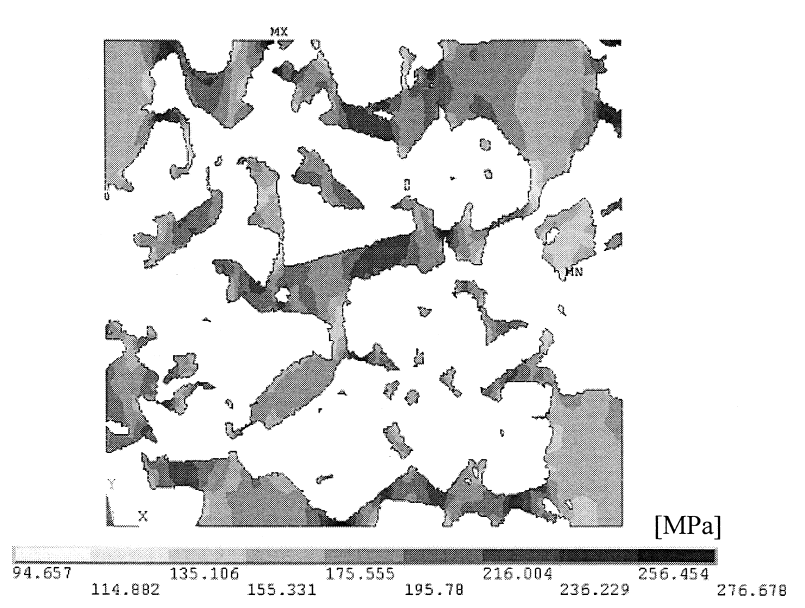

Fig. 12. Archiving the stress and strain information by specifying the segmented region.

\subsection{Estimate of Fracture Strength for Sintered Ore Specimen}

The sintered ore specimen is composed of many textures. Consider that the specimen consists of two types of texture: the porous hematite (phase-I) and the slag-hematite complex (phase-2). In order to generate the theoretical model, representative of this virtual specimen, the phase-field method $^{13,14)}$ was utilized. First, the spatial distribution of two phases was estimated and each region respectively for phase-I and -II was discretized into the theoretical model. Figure 13 depicted the generated theoretical model for the sintered-ore specimen under uniform compression. Instead of the crushing test, the stress and strain analyses were made by applying the uniform strain to this model. Figure 14 depicted a typical strain distribution in the specimen. Once the maximum strain is obtained by archiving the calculated strain distribution, the fracture stress is deduced in the similar way to the estimate of the fracture stress for each texture. Figure 15 showed the variation of fracture stress with increasing the volume fraction of phase-I or porous hematite. With a little addition of phase-1 with lower texture strength, the fracture strength of the whole two-phase specimen is reduced. The lowest fracture strength of this two-phase specimen reaches to about $150 \mathrm{MPa}$. This converging strength is just corresponding to the fracture stress of single phase of porous hematite. 
Table 3. Estimate of fracture strength for each texture.

\begin{tabular}{|l|c|l|c|}
\hline Texture & Limit of Strain & \multicolumn{1}{|c|}{$\begin{array}{c}\text { Position with } \\
\text { Maximum Strain }\end{array}$} & $\begin{array}{c}\text { Fracture Stress } \\
(\mathrm{MPa})\end{array}$ \\
\hline Porous Hematite & 0.002 & - Near largest pore & 155 \\
\hline $\begin{array}{l}\text { Acicular } \\
\text { Calcium Ferrite }\end{array}$ & 0.002 & $\begin{array}{l}\text { - In matrix phase with lower } \\
\text { strength }\end{array}$ & 29 \\
\hline $\begin{array}{l}\text { Slag-Hematite } \\
\text { Complex }\end{array}$ & 0.002 & - In slag & 234 \\
\hline
\end{tabular}

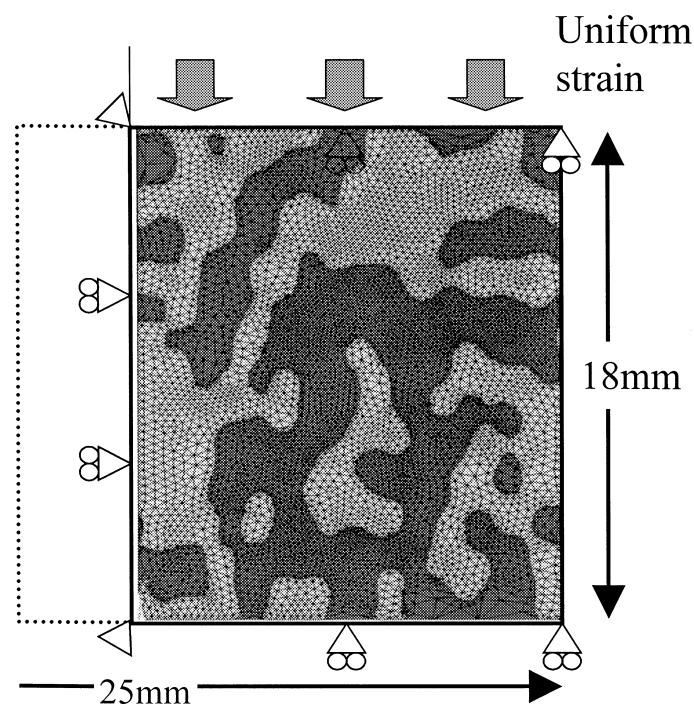

Fig. 13. Macro-model for the crushing test of sintered ore specimens.

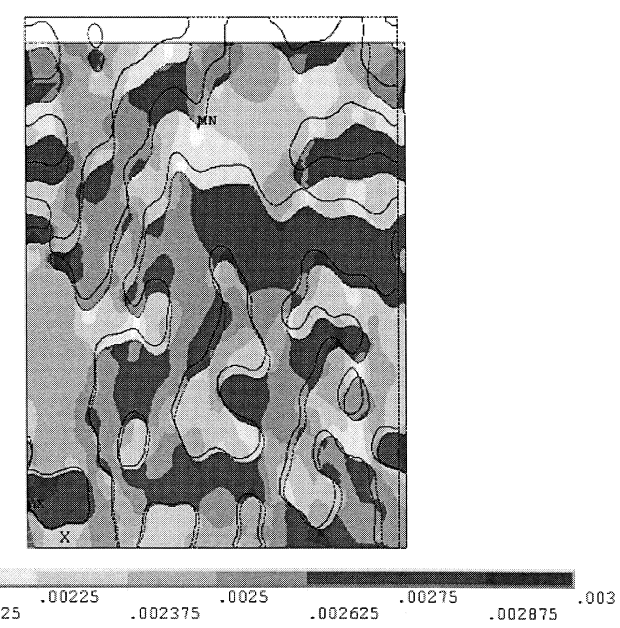

Fig. 14. Strain distribution of the deformed specimen under uniformly applied compressive strain.

\section{Conclusion}

The sintered ore is a porous medium, which has various size of pores and porosities and consists of various textures in microstructure. Its mechanical response is important to improve the total integrity and reliability of sintered ores in the furnace. The real microstructure modeling is effective to provide the theoretical approach to construct the accurate model for stiffness and strength evaluation on the sintered ore. For each constituent texture, its stiffness and fracture strength can be estimated with direct consideration of its

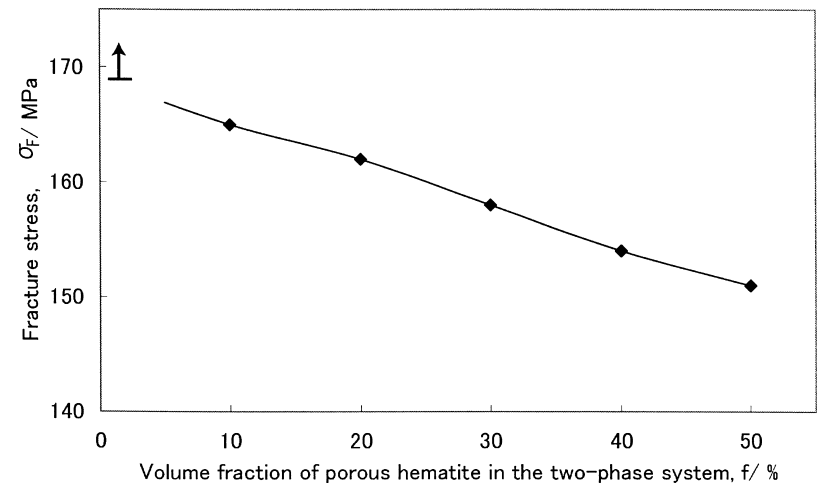

Fig. 15. Sensitivity of fracture stress to the composition of slag to hematite.

characteristic microstructure. The homogenized stiffness, calculated by this theoretical model, becomes equivalent to the elastic properties for each texture. Then, using these data of homogenized stiffness, the mechanical properties might well be designed for optimum combination of textures in the sintered ore. Furthermore, since the fracture strength can be estimated by the present approach for each texture, optimum design of fracture strength is to be carried out to search for the optimum geometry with demanded strength.

\section{Acknowledgments}

This study is financially supported in part by the national project from MEXT.

\section{REFERENCES}

1) M. Naito, A. Okamoto, K. Yamaguchi, T. Yamaguchi and Y. Inoue: Tetsu-to-Hagané, 87 (2001), 357.

2) A. Kasai, Y. Matsui, F. Noma, H. Iwakiri and M. Shimizu: Tetsu-toHagané, 87 (2001), 313.

3) F. Tsumori, T. Aizawa and J. Kihara: Jpn. J. Powder Metall., 43 (1996), 1165.

4) F. Tsumori, T. Aizawa and J. Kihara: Sintering Technol., (1996), 29.

5) F. Tsumori and T. Aizawa: Mater. Sci. Eng., A285 (2000), 357.

6) T. Aizawa, Y. Prawoto and F. Tsumori: J. Comp. Appl. Math., 149 (2002), 307.

7) Y. Prawato and T. Aizawa: Practical Failure Analysis, Vol. 2, ASM International, Materials Park, OH, (2002), 68.

8) T. Aizawa and Y. Suwa: Forum Maintenology, 1 (2003), 44

9) M. P. Bendsoe and N. Kikuchi: Comp. Meth. Appl. Mech. Eng., 71 (1988), 197.

10) B. Hassani and E. Hinton: Compos. Struct., 69 (1998), 719.

11) K. Terada and N. Kikuchi: Introduction to Homogenization Theory, Maruzen, Tokyo, (2003).

12) M. Asada and Y. Onishi: Tetsu-to-Hagané, 69 (1983), 98

13) D. Fan, C. Geng and L-Q. Chen: Acta Mater., 45 (1997), 1115.

14) Y. Suwa and Y. Saito: Mater. Trans., 44 (2003), 2245. 